a group at the Institut für Theoretische und Angewandte Physik (ITAP) of Stuttgart University carried out a demonstration run with over five billion particles. With 512 processing nodes and a total memory of $256 \mathrm{~GB}$, the program IMD (ITAP Molecular Dynamics) performed a simulation with 5,180,116,000 particles.

The atoms, interacting via LennardJones potentials, were placed in a cubic fcc lattice cell with an edge length of 1540 atom distances or $0.42 \mu \mathrm{m}$ (for $\mathrm{Al}$ ). This means that the simulations are arriving at scales comparable to the wavelength of visible light and to the width of the conductor paths of the computer. Six time steps were carried out and took a computer time of $2,328 \mathrm{~s}$. According to the researchers, 223 time steps would require about a day, a typical simulation more than a month. The real challenge, they said, is the output data which cannot be stored directly but has to be analyzed parallel to the simulation.

IMD is a software package designed to perform classical molecular dynamics simulations on massively parallel computers. The interactions are not limited to pair potentials. The program permits embedded atom potentials, three-body potentials, and anisotropic Gay-Berne-potentials. In addition to the common thermodynamic ensembles, a number of boundary conditions are implemented enabling the program to deform a sample, to stress load it, or to simulate shock waves.

\section{AFM Applied in Molecular-Scale Flattening of DAST Crystals}

Due to their nonlinear optical properties, certain organic crystals are useful in applications such as ultrahigh-speed signal detection and integrated circuit diagnosis. While the polishing of these soft organic crystals is required for optimizing light transmittance, the usual polishing and cutting techniques are often insufficient for flattening these materials. A research group from the Institute for Chemical Reaction Science at Tohoku University and the Intelligent Cosmos Research Institute has invented a molecular-scale polishing method utilizing atomic-force microscopy (AFM). As reported in the August 1 issue of Optics Letters, ion pairs of the 4-dimethylamino-N-methyl-4-stilbazolium tosylate (DAST) crystal may be removed by applying some force to the AFM cantilever tip.

Single DAST crystals were grown from seed crystals by lowering the temperature of a solution saturated with DAST. The flattening of the (001) surface was performed by the scanning action of the AFM. "When the force applied to an AFM tip is larger than that of the interlayer bond, the layer(s) that are exposed on the surface will be removed," the scientists reported. An approximately $10 \mathrm{nN}$ force was applied to the tip (with a tip curvature of $20 \mathrm{~nm}$ ) during the scan. Three stepped terraces appeared after the first five scans, and another five after eight scans. The average step height was approximately $0.9 \mathrm{~nm}$. This is a good agreement with the interlayer distance of $0.893 \mathrm{~nm}$. The scientists generated a terrace area of 250,000 $\mathrm{nm}^{2}$. A few existing terrace steps are not expected to contribute significantly to optical transmission loss.

A friction-force microscope (FFM) was used for characterizing the resulting DAST crystal. Topography photos indicate that terrace regions are indeed very flat, with a roughness of $0.12 \mathrm{~nm} \mathrm{rms}$. These results show that the AFM may be used as a polishing tool and that this technique shows promise for other organic crystals similar to DAST.

JUNE LAU

\section{Minimization of Diffuse Scattering Reduces Resistivity of Thin Copper Films}

Low resistivity values of $2-2.2 \mu \Omega \mathrm{cm}$ have been observed for copper films as thin as $30-40 \mathrm{~nm}$, comparable to the mean free path $(\mathrm{mfp})$ of electrons in copper. A team of researchers at Rensselaer Polytechnic Institute (RPI) reports that the deposition of copper thin films in an argon atmosphere containing $3 \mathrm{vol} \%$ hydrogen followed by a low temperature anneal considerably improves the conductivity of the films.

As semiconductor devices continue to shrink, the dimensions of the interconnect metals reach values comparable to the $\mathrm{mfp}$ of electrons. This results in a drastic increase in resistivity, which is attributed to diffuse electron scattering by surfaces. Passivation or tailoring of the surfaces that results in an increase of the elastic scattering component should therefore decrease the resistivity.

"Our experiments were designed to find conditions that may lead to a passivation of surfaces and thereby decrease the resistivity of thin copper films $(30-50 \mathrm{~nm})$. Earlier studies by Ficalora have indicated that sputter-depositing $\mathrm{Cu}$ in hydrogen lowered the as-deposited resistivity of $\mathrm{Cu}$ films that were greater than $100 \mathrm{~nm}$ in thickness. Ficalora has also shown that electromigration testing in hygrogen ambients caused significant improvements in the electromigration lifetimes of $\mathrm{Au}$ interconnects," said S.P. Murarka, professor of materials science and engineering at RPI.

As reported in the September issue of
Electrochemical and Solid-State Letters, the scientists investigated films between 10 and $200 \mathrm{~nm}$ in thickness prepared by dc magnetron sputter deposition of copper. Substrates were $p$-type silicon with a uniform $50 \mathrm{~nm}$ thermally oxidized layer. The copper depositions were carried out in pure argon or in argon with $3 \mathrm{vol} \%$ hydrogen and annealed in high vacuum or in an argon atmosphere with 3\% hydrogen at $300^{\circ} \mathrm{C}$. The films that were prepared in the hydrogen-containing atmosphere showed a resistivity that was on average $10 \%$ lower than for films prepared in pure argon, and the resistivity at $39 \mathrm{~nm}$ thickness was estimated to be $2-2.2 \mu \Omega \mathrm{cm}$. The resistivity of the films deposited in pure argon could be decreased by $10-30 \%$ by annealing in vacuum or $\mathrm{Ar} / \mathrm{H}_{2}$. The use of $\mathrm{Ar} / \mathrm{H}_{2}$ gave $2-5 \%$ lower resistivity values than the vacuum treatment.

"The role of hydrogen in affecting the resistivity of as-deposited films is not clear yet," said Anupama Mallikarjunan, a graduate student in Murarka's group. "It seems to passivate the electron-scattering defects including surfaces, enhancing the overall mobility across the film."

Another factor, according to the researchers, could be that hydrogen scavenges the chamber of impurities like oxygen, water, and nitrogen. Another group at RPI is currently working on in situ experiments in ultrahigh vacuum chambers to address these issues.

CORA LIND

\section{High-Valence Cations Improve Hydration Resistance of MgO-CaO Materials}

Improving the hydration resistance of magnesite and dolomite materials has important implications for the ceramics industry, particularly in the refractories sector where $\mathrm{MgO}-\mathrm{CaO}$ bricks are often used to line basic open hearth furnaces. Research reported in the July issue of the Journal of the American Ceramic Society reveals that the addition of tri- and tetravalent cations in small proportions can improve hydration resistance by producing vacancies in solid solutions of $\mathrm{CaO}$ and $\mathrm{MgO}$, and by substituting cations that are less prone to hydration.

Researchers at the East China University of Science and Technology in Shanghai and the Anshan Institute of Iron and Steel Technology pressed and fired high-density briquettes of flotation magnesite and dolomite doped with $0.5 \%$ (cation basis) of various monovalent to tetravalent cations. Dopants included $\mathrm{NaF}, \mathrm{NaCl}, \mathrm{CaF}_{2}$, $\mathrm{MgCl}_{2}, \mathrm{Al}_{2} \mathrm{O}_{3}, \mathrm{Cr}_{2} \mathrm{O}_{3}, \mathrm{Fe}_{2} \mathrm{O}_{3}, \mathrm{TiO}_{2}$, and $\mathrm{ZrO}_{2}$. Briquettes fabricated from analytically pure $\mathrm{MgO}$ and $\mathrm{CaO}$ were included 
for control purposes. After grinding the calcined samples into powders, hydration tests were performed by placing the powders into a constant temperature and humidity (96\%) chamber for up to four hours. The hydration rate was calculated as the ratio of the weight gain after hydration to the original sample weight.

Hydration rates of $0.5 \%$ to $1.8 \%$ were obtained, with the lower hydration rates generally corresponding to a higher valency of the dopant. The researchers attribute these results to two phenomena. $\mathrm{Ca}^{2+}$ and $\mathrm{Mg}^{2+}$ vacancies formed by reactions with the dopants increase the surface energy and enhance the sintering process, leading to more dense powders. Also, the preferential formation of solid solutions involving high-valency dopants (such as $\mathrm{Ti}^{4+}$ ) with the more-easily hydrated $\mathrm{CaO}$ phase decreases the $\mathrm{Ca}^{2+}$ concentration, reducing the tendency to hydrate.

TIM PALUCKA

\section{Alex Zunger to Receive TMS 2001 John Bardeen Award}

Alex Zunger, NREL's Research Fellow and leader of the Solid State Theory group (Basic Sciences Center), is the recipient of the John Bardeen Award for the year 2001, presented by the Minerals, Metals, and Materials Society (TMS). He is cited for "his seminal contributions to the theoretical understanding and prediction of 'spontaneous ordering,' phase-stability, and electronic properties of semiconductor alloys; for the impact that this work has had on experimental studies of electronic materials, and for his continued leadership in the field.

This work centers around Zunger's pioneering seminal theoretical studies of "spontaneous ordering" in semiconductor alloys - a phenomenon whereby instead of appearing randomly, the atomic constituents of alloys (e.g., gallium and indium in gallium phosphide-indium phosphide) tend to order spatially in geometric arrangements that alter the electronic, transport, and optical properties of the material creating new, technologically significant functions. A more detailed description of this field appears in the group's web site at www.sst.nrel. gov/topics/alloy_order.html.

Zunger said that his work on this theoretical problem was done in collaboration with his postdoctoral fellows since 1983: J. Bernard, D. Laks, K. Mader, R. Magri, J.L. Martins, T. Mattila, A. Mbaye, R. Osorio, V. Ozolins, G.P. Srivastava, and D. Wood, as well as with his NREL associates L. Ferreira, S. Froyen, S.H.Wei, and S.B. Zhang. Zunger's other areas of interest include photovoltaic materials, the theory of semiconductor quantum-dots, and the theoretical design and predictions of stable crystal structures and phase-diagrams.

This award will be presented formally to Zunger at the 130th Annual TMS meeting on February 13, 2001, in New Orleans.

\section{Peppas Receives General Electric Senior Research Award}

Nicholas A. Peppas, the Showalter Distinguished Professor of Chemical and Biomedical Engineering in the School of Chemical Engineering at Purdue University, has been presented with the General Electric Senior Research Award at an awards ceremony held at the annual conference of the American Society for Engineering Education in St. Louis on June 21. He has been honored for fundamental and prolific contributions to polymer engineering and science, biomedical engineering, and pharmaceutical engineering. These contributions have provided insight into numerous engineering processes and applications and led to the development of new biomaterials and medical devices, including artificial vocal cords, contact lenses, linings for artificial hearts, artificial cartilage, a wide range of drug delivery devices, and the recently commercialized oral insulin delivery systems. Peppas was appointed to his current position in 1993, and has served on the faculty at Purdue since 1976.

\section{Johnson and MacChesney Receive International Ceramics Prize}

Scientists David Johnson and John MacChesney of Lucent's Bell Labs have received the International Ceramics Prize 2000 from the Academy of Ceramics at the Forum 2000 conference held in Maiori, Italy, in June. The award, which recognizes important contributions to ceramics research, cites MacChesney and Johnson's "outstanding contribution to sol-gel technology and its industrial application in the area of optical communications."

Johnson holds a PhD degree in ceramic science from Pennsylvania State University. He is also an adjunct professor of materials science at Stevens Institute of Technology, Hoboken, NJ. He is a Fellow of the American Ceramic Society and a member of the National Academy of Engineering, Materials Research Society, North American Thermal Analysis Society, American Association for the Advancement of Science, American Society of Materials International, and American Society for Testing Materials.
MacChesney holds a PhD degree from Pennsylvania State University and is an adjunct professor at Brown and Rutgers Universities, as well as the Kwangju Institute of Science and Technology in Korea. He was elected to the National Academy of Engineering in 1985. He is a Fellow of the American Ceramic Society.

\section{Nishizawa Receives IEEE Edison Medal}

Jun-Ichi Nishizawa, president of Iwate Prefectural University in Japan, has received the 2000 Institute of Electrical and Electronics Engineers (IEEE) Edison Medal at the annual IEEE Honors Ceremony on June 24 in Vancouver, BC, Canada. He is honored "for contributions to the materials science and technology and the invention of the static induction transistor."

Nishizawa has made significant contributions to materials science and technology, fiber optics, optical communications, and other areas. Early in his career, he developed the static induction transistor and the static induction thyristor (SIT) for high-power and high-frequency devices. To enhance the SIT's performance, Nishizawa established the Semiconductor Research Institute in Tohoku University, Japan, where he has been director since 1968. Among his contributions are work on the development of such semiconductor devices as the pin diode and photodiode, and the npin drift transistor.

Nishizawa is a Life Fellow of IEEE and a member of the Materials Research Society. He received his $\mathrm{BS}$ and $\mathrm{PhD}$ degrees from Tohoku University.

\section{Alexis Clare Receives 2000 Gottardi Prize}

Alexis G. Clare, associate professor of glass science in the School of Ceramic Engineering and Materials Science, has been awarded the Gottardi Prize by the International Commission on Glass (ICG) "for outstanding achievement in the field of glass research and development, teaching, writing, management, or commerce." She received the award during ICG's annual meeting in May in Amsterdam, The Netherlands.

Clare is director of the Alfred satellite of the National Science Foundation's Industry-University Center for Biosurfaces. She is a member and Fellow of the American Ceramic Society and the Society of Glass Technology. She received her BS degree in chemical physics and her $\mathrm{PhD}$ degree in physics, both from the University of Reading, England. 